\title{
Development of Nursing Informatics in Mainland China: A Bibliometric Analysis
}

\author{
Jialin LIU ${ }^{\mathrm{a}, 1}$, Siru LIU ${ }^{\mathrm{b}}$, Tao ZHENG ${ }^{\mathrm{a}}$ and Jinbo FANG ${ }^{\mathrm{c}}$ \\ ${ }^{a}$ Department of Medical Informatics, West China Hospital, Sichuan University, China \\ ${ }^{\mathrm{b}}$ Department of Biomedical Informatics, University of Utah, Salt Lake City, Utah, USA \\ ${ }^{\mathrm{c}}$ West China School of Nursing, West China Hospital, Sichuan University, China
}

\begin{abstract}
This study aimed to understand the status of nursing informatics in Mainland China. Articles on nursing informatics, published between 2009 and 2018, were retrieved from the CNKI (China National Knowledge Infrastructure) database. A total of 51 papers were identified and analyzed. The journals, annual publications, and co-occurrence of keywords were analyzed with the bibliometric analysis. The result will help us better understand the nursing informatics research in mainland China.
\end{abstract}

Keywords. Nursing informatics, bibliometric analysis, China

\section{Introduction}

China is the second-largest economy in the world with a population of nearly 1.4 billion. There is a vast health information technology market and significant investment in health information systems. However, the contribution of Chinese scholars in the field of nursing informatics to global society is very limited. The study aims to map the research output of nursing informatics in mainland China and understand the current status of nursing informatics by bibliometric analysis.

\section{Method}

A literature search was performed in the CNKI database for articles period from January 1, 2009, to December 31, 2018. The CNKI is the largest Chinese database in the world and offers the latest scientific and technical information from China [1]. We used the following topic term: "nursing informatics" or title: "nursing informatics" to search. To facilitate cross-checking, all data were independently collected by two authors and downloaded in text format. Only articles and reviews on nursing informatics were included in the analysis. We use Microsoft Excel 2016 for data extraction and analysis. The CNKI tool was used to create the keyword co-occurrence matrix.

\footnotetext{
${ }^{1}$ Corresponding Author, Jialin Liu, Department of Medical Informatics, West China Medical School, Sichuan University, Chengdu, Sichuan, China; E-mail: dlj18@163.com.
} 


\section{Results}

From the CNKI database, 58 articles were selected for preliminary analysis. After reviewing the title and the full text, seven irrelevant articles to the study themes $(11.54 \%)$ were excluded. Finally, 51 articles were identified for analysis. Of these 51 articles, 17 were original articles (33.33\%) and 34 articles were reviews (66.67\%).

\subsection{Journals Publishing on Nursing Informatics}

In total, 51 articles were published in 21 different journals. The top five journals in the field of producing articles published 38 articles (68.63\%). There were 14 different journals with only one article published. Only four articles were published in three different Chinese core journals (Chinese Journal of Nursing, Journal of Nursing Training, and Journal of Southern Medical University).

\subsection{Co-occurrence of Author Keywords Analysis}

From 2009 to 2018, a total of 154 author keywords were included in 51 articles. The cooccurrence matrix spreadsheets of keywords were constructed by the CNKI tool (Figure 1). The most common keywords are "nursing informatics," "nursing," "education," "nursing education" and "information literature."
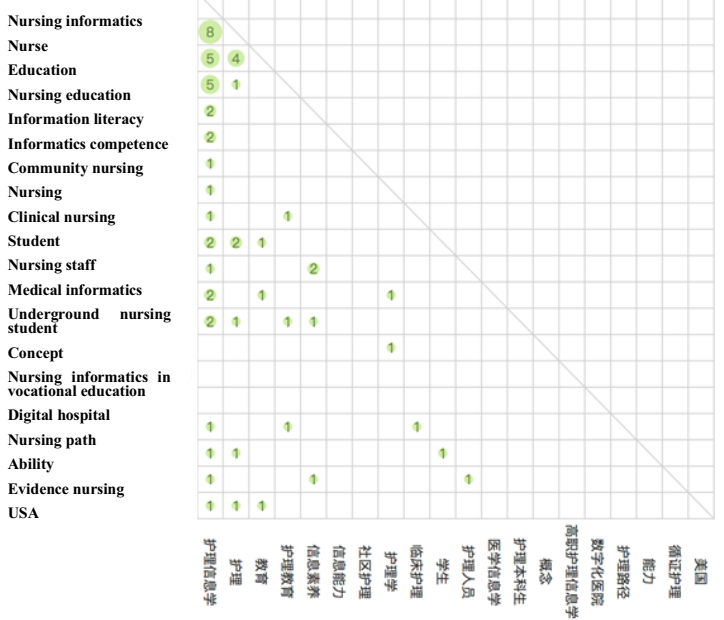

Figure 1. Keywords co=occurrence matrix.

\section{Conclusions}

There is little research on the theoretical and technical aspects of nursing informatics in Chinese articles. These results will help us understand the research evolution of nursing informatics in China over a ten-year period. 


\section{References}

[1] http://online.ebiblioteka.ru/help/CNKI_Flyer.pdf, accessed on January 21, 2019 